\title{
Applying interior points methods for the radiotherapy planning problem in a fuzzy approach background
}

\author{
Jackeline del Carmen Huaccha Neyra ${ }^{1}$ \\ IMECC/UNICAMP, SP \\ Aurelio Ribeiro Leite de Oliveira ${ }^{2}$ \\ IMECC/UNICAMP, SP
}

\begin{abstract}
A Radiation Therapy Design Problem consists in minimize the total radiation dosage at the patient. In this work, the dosage values are represented by fuzzy numbers. The transition from healthy tissue to tumour cells is continuous and the surprise function is adopted to model it. We propose to solve this problem by a specially tailored Primal-Dual Interior Point Method and present numerical experiments with real world large-scale problems.
\end{abstract}

Keywords. Interior Point Methods, Radiation Therapy, Fuzzy Optimization

\section{Introduction}

Radiation therapy is a technique used for treating cancer patients with ionic radiation. Its aim is to deliver a dose of radiation in order to eliminate tumour cells avoiding, as much as possible, the nearby healthy tissues and critical organs. This process is made taking a CT-scan (Computed Tomography) to obtain images of the patient.

Generally, in the literature, there is no agreement on the function objective. This function should minimize total radiation, maximize minimum tumor dosage, minimize radiation to critical structures, etc. In practice, is difficult to ensure that with an exact quantity of dose it is possible to achieve the purposes of the radiation therapy, since the oncologist can vary the dose distribution according to his expertise and tolerances of each region. Thus, in 2001, Lodwick et al. [5] presented three approaches to use fuzzy optimization in the radiation therapy problems where the fuzziness appears on the right-hand side. One of these approaches considers the dosage values as fuzzy numbers and uses the theory of surprise functions, developed by Neumaier, in 2003, [6], to translate the fuzzy constraints into non-linear programming problem.

Fuzzy optimization is used to ensure that the constraints have a high membership degree.

In this work we will combine these two concepts, where the fuzzyness will be considered on the bounds of the desired dose of radiation as being triangular fuzzy numbers. Another types of fuzziness, in which more general cases are considered, can be seen in [3], [7], [8], [10].

\footnotetext{
1 jacky.157.93@gmail.com

2aurelio@ime.unicamp.br
} 


\section{Problem Formulation}

The mathematical representation correspondent to fuzzy optimization radiotherapy problem is $($ see $[4])$ :

$$
\begin{array}{ll}
\min & c^{T} x \\
\text { s.t } & A x \leq \tilde{b} \\
& 0 \leq x
\end{array}
$$

where, considering that the images of the patient are discretized in $T P$ pixels and the radiation delivered is shaped by $N$ beamlets,

$A \in \mathbb{R}^{T P \times N}$ : is the attenuation matrix that represents how one unit of radiation intensity in each beamlet is deposited in pixels, $A=\left[\begin{array}{lll}A_{T} & A_{C} & A_{B}\end{array}\right]^{T}$ in which $A_{T}$ is the matrix corresponds to tumour pixels, $A_{C}$ corresponds to critical organs and $A_{B}$ corresponds to healthy tissues surrounding the tumour,

$\tilde{b} \in \mathbb{R}^{T P}$ : is a vector with the desired dose. In this approach, the components are triangular fuzzy numbers,

$c \in \mathbb{R}^{T P}:$ is a vector chosen appropriately according to our goals,

$x \in \mathbb{R}^{N}$ : variables that represent the beamlet intensities.

A fuzzy number [9], $\tilde{u}$, is a special type of fuzzy set where the membership function $\mu$ is defined on $\mathbb{R}$, that is, $\mu: \mathbb{R} \rightarrow[0,1], \mu(x)$ measures the degree of belonging of $x$ in $\tilde{u}$.

The $\alpha$-cut of the fuzzy number $\tilde{u}$, denoted as $[\tilde{u}]_{\alpha}=\{x \in \mathbb{R}: \mu(x) \geq \alpha\}$ is a non-empty, bounded and closed interval for all $\alpha \in(0,1]$. Taking $u_{L}^{\alpha}$ and $u_{R}^{\alpha}$ as the extremes of the interval, a fuzzy number $\tilde{u}$ can be defined by the $\alpha$-cut, $[\tilde{u}]_{\alpha}=\left[u_{L}^{\alpha}, u_{R}^{\alpha}\right]$.

Thus, a triangular fuzzy number $\tilde{u}=\left(u_{1} u u_{2}\right)$ defined by the $\alpha$-cut is the interval

$$
[\tilde{u}]_{\alpha}=\left[u_{1}+\alpha\left(u-u_{1}\right), u_{2}+\alpha\left(u-u_{2}\right)\right] \text { for all } \alpha \in(0,1] .
$$

As fuzzy numbers are fuzzy sets, in (1) each component of $A x$ has a degree of belonging to a subset of $\mathbb{R}$, the respective component of $\tilde{b}$. This case will be treated bellow using the surprise function method (see [4]) that searches the best solution in the constraints for each $i=1, \ldots, T P$

$$
\sum_{j=1}^{N} a_{i j} x_{j} \leq \tilde{b}_{i} \Longleftrightarrow\left(\sum_{j=1}^{N} a_{i j} x_{j}=\tilde{\xi}_{i}, \quad \mu_{i}\left(\xi_{i}\right)=\operatorname{pos}\left(\tilde{b}_{i} \geq \tilde{\xi}_{i}\right)\right)
$$

where $\mu$ is the membership function and the surprise function is obtained from $\mu$ by $s(\xi)=$ $\left(\frac{1}{\mu(\xi)}-1\right)^{2}$. The surprise function, so defined, measures the violation of constraints, therefore combining these violations, the formulation of fuzzy optimization using surprise functions obtained is $($ see $[6])$ :

$$
\begin{array}{ll}
\min & \sum_{i=1}^{T P} s_{i}\left(\sum_{j=1}^{N} a_{i j} x_{j}\right) \\
\text { s.t. } & 0 \leq x \leq U
\end{array}
$$

where the objective function minimizes the total dosage. The single constraint guarantees that the radiation of the beams be bounded.

The model (3) can be modified in order to ensure a good quality of solutions. We will add the following constraints: 
- Taking into account the relation (2), $\xi$ will be considered as a variable that satisfies: $A x=\xi$.

- $\xi$ being a variable must satisfy $\xi \leq \tilde{b}=\left(\begin{array}{lll}b_{1} & b & b_{2}\end{array}\right)$ (triangular fuzzy number). Out of the interval $\left(b_{1}, b_{2}\right)$ the surprise functions take high values. Then $b_{1} \leq \xi \leq b_{2}$ guarantees that the surprise functions will be small and, therefore, $f(x)$ be minimized.

The above analysis allows us to define an improved formulation of the problem:

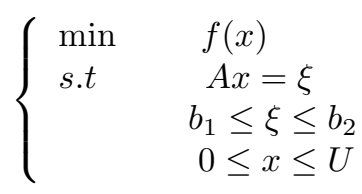

\section{The interior point method}

Applying the interior point method on formulation (4) and adding the variables $v, z_{1}, z_{2} \geq 0$, (4) becomes

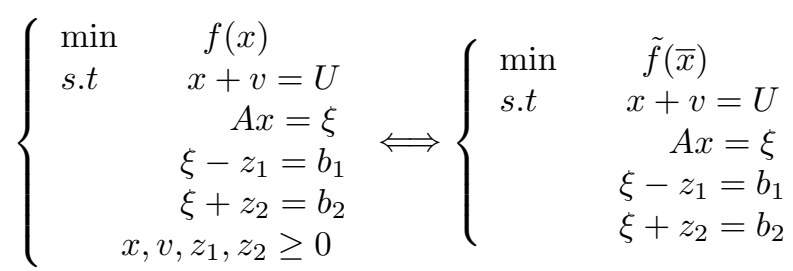

where

$$
\tilde{f}(\bar{x})=f(x)-\gamma_{x} \sum_{i=1}^{N} \ln x_{i}-\gamma_{v} \sum_{i=1}^{N} \ln v_{i}-\gamma_{z_{1}} \sum_{i=1}^{T P} \ln z_{i}^{1}-\gamma_{z_{2}} \sum_{i=1}^{T P} \ln z_{i}^{2},
$$

$\bar{x}=\left(x, v, z_{1}, z_{2}\right)$ and the non-negativity of $\bar{x}$ is implicit in the logarithm function.

Let the Lagrangean function of $\tilde{f}$ be

$$
\mathcal{L}(\bar{x} ; \bar{y})=\tilde{f}(x)-y^{T}(x+v-U)-w_{1}^{T}\left(\xi-z_{1}-b_{1}\right)-w_{2}^{T}\left(\xi+z_{2}-b_{2}\right)-q^{T}(A x-\xi)
$$

where $\bar{y}=\left(y, w_{1}, w_{2}, q\right)$ is the non-negative dual variable of the formulation (4).

Computing the gradient of $\mathcal{L}$ :

$$
\begin{aligned}
\nabla_{x} \mathcal{L}(\bar{x} ; \bar{y}) & =\nabla f(x)-\gamma_{x} X^{-1} e-y-A^{T} q \\
\nabla_{\xi} \mathcal{L}(\bar{x} ; \bar{y}) & =-w_{1}-w_{2}+q \\
\nabla_{v} \mathcal{L}(\bar{x} ; \bar{y}) & =-\gamma_{v} V^{-1} e-y \\
\nabla_{z_{1}} \mathcal{L}(\bar{x} ; \bar{y}) & =-\gamma_{z_{1}} Z_{2}^{-1} e+w_{1} \\
\nabla_{z_{2}} \mathcal{L}(\bar{x} ; \bar{y}) & =-\gamma_{z_{2}} Z_{2}^{-1} e-w_{2} \\
\nabla_{y} \mathcal{L}(\bar{x} ; \bar{y}) & =-(x+v-U) \\
\nabla_{w_{1}} \mathcal{L}(\bar{x} ; \bar{y}) & =-\left(\xi-z_{1}-b_{1}\right) \\
\nabla_{w_{2}} \mathcal{L}(\bar{x} ; \bar{y}) & =-\left(\xi+z_{2}-b_{2}\right) \\
\nabla_{q} \mathcal{L}(\bar{x} ; \bar{y}) & =-(A x-\xi)
\end{aligned}
$$

where $X, V, Z_{1}$ and $Z_{2}$ are diagonal matrix formed by the components of $x, v, z_{1}$ and $z_{2}$, respectively, and $e$ is the vector of $1^{\prime} s$. Let $p, s, t_{1}$ and $t_{2}$ be the complementary (non-negative) variables 
such that

$$
\begin{aligned}
p=\gamma_{x} X^{-1} & \Longleftrightarrow X P e=\gamma_{x} e, \\
s=\gamma_{v} V^{-1} & \Longleftrightarrow V S e=\gamma_{v} e, \\
t_{1}=\gamma_{z_{1}} Z_{1}^{-1} & \Longleftrightarrow Z_{1} T_{1} e=\gamma_{z_{1}} e, \\
t_{2}=\gamma_{z_{2}} Z_{2}^{-1} & \Longleftrightarrow Z_{2} T_{2} e=\gamma_{z_{2}} e .
\end{aligned}
$$

Replacing on gradient of $\mathcal{L}$, in (6), we obtain the function $F$

$$
F=\left(\begin{array}{c}
\nabla f(x)-p-y-A^{T} q \\
-w_{1}-w_{2}+q \\
y+s \\
-t_{1}+w_{1} \\
t_{2}+w_{2} \\
x+v-U \\
\xi-z_{1}-b_{1} \\
\xi+z_{2}-b_{2} \\
A x-\xi \\
X P e-\gamma_{x} e \\
V S e-\gamma_{v} e \\
T_{1} Z_{1} e-\gamma_{z_{1}} e \\
T_{2} Z_{2} e-\gamma_{z_{2}} e
\end{array}\right)
$$

Applying the Newton method to the equation $F=0$, we obtain

$$
\left(\begin{array}{ccccccccccccc}
H & 0 & 0 & 0 & 0 & -I & 0 & 0 & -A^{T} & -I & 0 & 0 & 0 \\
0 & 0 & 0 & 0 & 0 & 0 & -I & -I & I & 0 & 0 & 0 & 0 \\
0 & 0 & 0 & 0 & 0 & I & 0 & 0 & 0 & 0 & I & 0 & 0 \\
0 & 0 & 0 & 0 & 0 & 0 & I & 0 & 0 & 0 & 0 & -I & 0 \\
0 & 0 & 0 & 0 & 0 & 0 & 0 & I & 0 & 0 & 0 & 0 & I \\
I & 0 & I & 0 & 0 & 0 & 0 & 0 & 0 & 0 & 0 & 0 & 0 \\
0 & I & 0 & -I & 0 & 0 & 0 & 0 & 0 & 0 & 0 & 0 & 0 \\
0 & I & 0 & 0 & I & 0 & 0 & 0 & 0 & 0 & 0 & 0 & 0 \\
A & -I & 0 & 0 & 0 & 0 & 0 & 0 & 0 & 0 & 0 & 0 & 0 \\
P & 0 & 0 & 0 & 0 & 0 & 0 & 0 & 0 & X & 0 & 0 & 0 \\
0 & 0 & S & 0 & 0 & 0 & 0 & 0 & 0 & 0 & V & 0 & 0 \\
0 & 0 & 0 & T_{1} & 0 & 0 & 0 & 0 & 0 & 0 & 0 & Z_{1} & 0 \\
0 & 0 & 0 & 0 & T_{2} & 0 & 0 & 0 & 0 & 0 & 0 & 0 & Z_{2}
\end{array}\right) . \mathbf{d}=\mathbf{r}
$$

where $H$ is the $N \times N$ - Hessian matrix of $f, d=\left[\begin{array}{lll}d x d \xi d v d z_{1} d z_{2} d y d w_{1} d w_{2} d q d p d s d t_{1} d t_{2}\end{array}\right]^{T}$ and $\mathbf{r}=-F$

Solving (8) and denoting $X^{-1} P+V^{-1} S$ and $Z_{1}^{-1} T_{1}+Z_{2}^{-1} T_{2}$ as $D_{x}$ and $D_{z}$, respectively, we 
obtain

$$
\begin{aligned}
{\left[H+D_{x}+A^{T} D_{z} A\right] d x } & =r_{x} \\
d \xi & =A d x-r_{9} \\
d v & =r_{6}-d x \\
d z_{1} & =d \xi-r_{7} \\
d z_{2} & =r_{8}-d \xi \\
d p & =X^{-1}\left(r_{10}-P d x\right) \\
d s & =V^{-1}\left(r_{11}-S d x\right) \\
d t_{1} & =Z_{1}^{-1}\left(r_{12}-T_{1} d z_{1}\right) \\
d t_{2} & =Z_{2}^{-1}\left(r_{13}-T_{2} d z_{2}\right) \\
d q & =r_{2}+d w_{1}+d w_{2} \\
d y & =r_{3}-d s \\
d w_{1} & =r_{4}+d t_{1} \\
d w_{2} & =r_{5}-d t_{2}
\end{aligned}
$$

where

$$
\begin{gathered}
r_{x}:=r_{1}+A^{T}\left(r_{2}+r_{w_{1}}+r_{w_{2}}\right)+r_{3}+V^{-1}\left(S r_{6}-r_{11}\right)+X^{-1} r_{10} \\
r_{w_{1}}:=r_{4}+Z_{1}^{-1}\left[r_{12}+T_{1}\left(r_{7}+r_{9}\right)\right], \quad r_{w_{2}}:=r_{5}-Z_{2}^{-1}\left[r_{13}-T_{2}\left(r_{8}+r_{9}\right)\right]
\end{gathered}
$$

The system (9) allows us to compute the direction vectors. The main difficult is to compute $d x$. However, $D_{x}$ and $D_{z}$ are diagonal matrices and, in this case, defining, for each $i=1, \ldots, T P$, $M_{i}\left(\xi_{i}\right)=3 \mu_{i}\left(\xi_{i}\right)^{-4}-2 \mu_{i}\left(\xi_{i}\right)^{-3}$ and

$$
d_{H_{i}}=\left\{\begin{array}{cl}
\frac{2 M_{i}}{\left(b_{i}-b_{i}^{1}\right)^{2}}, & \text { if } \xi_{i} \in\left(b_{i}^{1}, b_{i}\right) \\
\frac{2 M_{i}}{\left(b_{i}^{2}-b_{i}\right)^{2}}, & \text { if } \xi_{i} \in\left(b_{i}, b_{i}^{2}\right) \\
0, & \text { otherwise }
\end{array}\right.
$$

$H=A^{T} D_{H} A$ and taking $D=D_{H}+D_{z}$. Therefore, to compute $d x$ the linear system $B d x=r_{x}$ is solved, with $B=A^{T} D A+D_{x}$ being a symmetric positive definite matrix.

\section{Computational Results}

The algorithm is tested in MATLAB for the first instance, Head-and-Neck-01, from the TROTS dataset [1], that has 9977 beamlets.

The tumour has 5096 pixels with dose $(24.543 .4748 .3)$, these values indicate that a dose of 43.47 will eliminate the tumour cells. The critical organ considered is spinal cord with 3529 pixels and dose ( $\left.\begin{array}{lll}0 & 22.8 & 38\end{array}\right)$, indicating that dose more than 38 are completely unacceptable. After 1000 iterations, we obtained solution $x$ with value objective function $f(x)=1.73662$ guaranteeing that the delivered dosage satisfies to radiation oncologist.

Moreover, the Erasmuns-iCycle solver (developed in [2]) considers all the critical organs and healthy tissue on the objective function and they are optimized taking into account different priorities. Then, the optimal solution provided in [1], solution $X$, has as value objective function (4) $f($ solution $X)=4938.867182 \times 10^{6}$.

The high difference between both solutions is because, with solution $X$, the radiation deliveries on the spinal cord is far from the value expected by the oncologist increasing the value of the surprise function and, therefore, the objective function. 


\section{Conclusion}

The approach proposed solves satisfactorily the problem attaining the ideal dosage to kill the tumour. Comparing with the solver, the solution obtained in this work is better because keeps minimal values of radiation for the critical organ, decreasing the risks and future complications.

\section{Acknowledgement}

To CAPES for the financial support.

\section{References}

[1] Breedveld, S. and Heijmen, B. TROTS - The Radiotherapy Optimisation TestSetA. https://www.erasmusmc.nl/en/cancer-institute/research/projects/trots-the-radiotherapyoptimisation-test-set

[2] Breedveld, S., van den Berg, B. and Heijmen, B. An interior-point implementation developed and tuned for radiation therapy treatment planning, Computational Optimization and Applications, 68:209-242, 2017. DOI: 10.1007/s10589-017-9919-4.

[3] Jamison, K. D. and Lodwick, W. A. Fuzzy linear programming using penalty methods, Fuzzy Sets and Systems, 119(1):97-110, 2001. DOI: 10.1016/S0165-0114(99)00082-2.

[4] Lodwick, W. A. and Bachman, K. A. Solving large-scale fuzzy and possibilistic optimization problems, Fuzzy Optimization and Decision Making, 4:257-278, 2005. DOI: 10.1007/s10700005-3663-4.

[5] Lodwick W. A., Neumaier A. and Newman F. Optimization Under Uncertainty Methods and Applications in Radiation Therapy, Proceedings 10th IEEE International Conference on Fuzzy Systems, 3:1219-1222, 2001.

[6] Neumaier, A. Fuzzy modeling in terms of surprise, Fuzzy Sets and Systems, 1(135): 21-38, 2003. DOI: 10.1016/S0165-0114(02)00248-8.

[7] Tanaka, H. and Assai, K. Fuzzy linear programming based on fuzzy functions, IFAC Proceedings Volumes, 14(2):785-790, 1981. DOI: 10.1016/S1474-6670(17)63578-X.

[8] Tanaka, H. and Assai, K. Fuzzy linear programming problems with fuzzy numbers, Fuzzy Sets and Systems, 13(1):1-10, 1984. DOI: 10.1016/0165-0114(84)90022-8.

[9] Zadeh L. A. Fuzzy sets, Information and Control, 8(3):338-353, 1965. DOI: 10.1016/S00199958(65)90241-X

[10] Zimmermann, H. -J. Fuzzy programming and linear programming with several objective functions, Fuzzy Sets and Systems, 1(1):45-55, 1978. DOI: 10.1016/0165-0114(78)90031-3. 